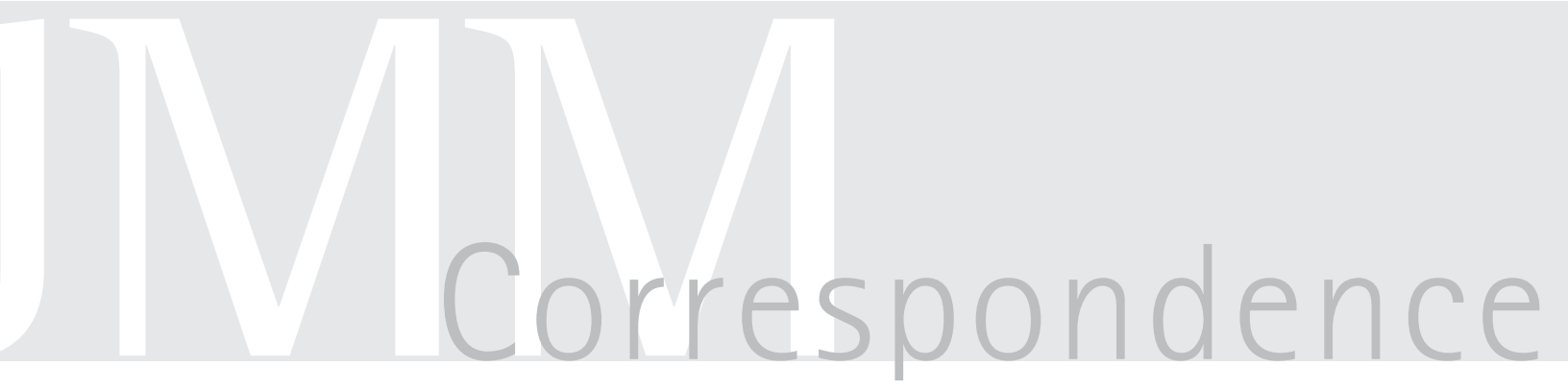

\title{
Misidentification of Brucella melitensis as Ochrobactrum anthropi by API 20NE
}

Brucella organisms have been misidentified as Moraxella phenylpyruvica by the API 20NE non-enteric identification system (bioMérieux), as Moraxella species by the MicroScan Negative COMBO type 5 system (Dade MicroScan) (Batchelor et al., 1992) and as Haemophilus influenzae biotype IV by the Haemophilus-Neisseria identification (HNID) panel (Dade MicroScan) (Barham et al., 1993). Ochrobactrum anthropi, formerly classified under CDC Group Vd, is an oxidasepositive, non-lactose-fermenting, Gramnegative bacillus of low virulence that occasionally causes human infection. Here, we report misidentification of Brucella melitensis by API 20NE as O. anthropi in a case of brucellosis and we highlight the importance of differentiation from other cases of pseudobacteraemia caused by $O$. anthropi.

On 18 May 2002, a blood culture gave a positive signal after $48 \mathrm{~h}$ incubation on the
BACTEC 9240 system. A slowly growing, oxidase-positive, Gram-negative bacillus failed to grow on MacConkey agar and was identified as O. anthropi in the API 20NE system, profile 1207004. This was thought to be of uncertain clinical relevance. However, because the patient was being investigated for possible endocarditis, repeat blood cultures were advised to help to assess significance. A further set of blood cultures sent on 23 May 2002 flagged positive after $48 \mathrm{~h}$ incubation. Gramnegative bacilli that failed to grow on MacConkey agar were isolated and identified as O. anthropi in the API 20NE system, profile 1201724. Further clinical history revealed that the patient's son, who had accompanied him on a trip to Cyprus, had been admitted to another hospital suffering from brucellosis. Subsequently, the patient's serum was tested for the presence of Brucella antibody. This test was positive and the reference laboratory confirmed acute Brucella infection by

Table 1. Confirmation of identification of blood culture isolates as Brucella melitensis

\begin{tabular}{|lc|}
\hline Characteristic & Result \\
\hline Growth characteristics: & \\
Urea & + \\
$\mathrm{H}_{2} \mathrm{~S}$ & - \\
$\mathrm{CO}_{2}$ & - \\
Basic fuschin $^{\star}$ & + \\
Thionin* & + \\
Detection of surface antigens with monospecific sera: & \\
$\mathrm{A}$ & + \\
$\mathrm{M}$ & - \\
Phages at routine test dilution: $\dagger$ & \\
Wb & $\mathrm{NL}$ \\
$\mathrm{Tb}$ & $\mathrm{NL}$ \\
$\mathrm{BK}$ & $\mathrm{CL}$ \\
$\mathrm{Fi}$ & $\mathrm{NL}$ \\
$\mathrm{R} / \mathrm{C}$ & $\mathrm{NL}$ \\
Detection of DNA by PCR & + \\
\hline
\end{tabular}

${ }^{\star}$ Tested at $20 \mu \mathrm{ml}^{-1}(1: 50000, \mathrm{v} / \mathrm{v})$.

$\dagger \mathrm{CL}$, Confluent lysis; NL, no lysis.

Brucella microagglutination test, complement fixation test, ELISA IgG and IgM. All blood culture isolates sent to a reference laboratory (Veterinary Laboratories Agency, Weybridge, Surrey, UK) were identified as Brucella melitensis (Table 1). The patient was treated for Brucella endocarditis with doxycycline and streptomycin.

On 10 September 2002, a Nigerian woman was admitted to the accident and emergency department with pyrexia, lower abdominal pain and vomiting for the previous $24 \mathrm{~h}$. A diagnosis of acute appendicitis was made. Appendectomy was performed and a gangrenous, ischaemic appendix was removed. A blood culture from the patient grew an oxidase-positive, non-motile, Gram-negative bacillus that failed to grow on MacConkey agar and was identified as $O$. anthropi by API 20NE, profile 1241145 . The patient's serum was tested negative for Brucella antibody and the reference laboratory at the Veterinary Laboratories Agency - Weybridge confirmed that the blood culture isolate from this patient was not Brucella. This isolate was sent to the Central Public Health Laboratory (Colindale, London, UK) and was confirmed as $O$. anthropi by fatty acid analysis. The patient recovered and was discharged home without antimicrobial therapy.

We have conducted a MedLine search to review all the literature on reported cases of O. anthropi infection. API $20 \mathrm{NE}$ was used for identification of 32 isolates of $O$. anthropi, 41 isolates were identified by using other methods and no microbiological method of identification was mentioned for 20 isolates. We found that cases of $O$. anthropi bacteraemia reported in the literature fell into two groups: those who made a full, uncomplicated recovery and those who had serious infections. Patients with pseudobacteraemia (El-Zimaity et al., 2001), catheter-associated sepsis (Cieslak et al., 1992) or bacteraemia due to intrinsic drug contamination (Ezzedine et al., 1994) recovered fully, some even without the use 
of antimicrobial therapy, and no relapses of O. anthropi bacteraemia occurred after removal of the source of infection. These cases reflect and confirm the low pathogenicity of this organism. The group of patients with severe disease required multiple antimicrobial therapy, as in cases of transplant-related infection (Chang et al., 1996), infective endocarditis (Saeed Mahmood et al., 2000) and other pyogenic infections (Yu et al., 1998). Our findings highlight the fact that $O$. anthropi, with its low virulence, should be differentiated from Brucella in patients presenting with severe disease manifested primarily as $O$. anthropi bacteraemia with no obvious focus of infection.

\section{Acknowledgements}

We would like to extend our gratitude to laboratory staff for their skilled testing of specimens and staff at both the Veterinary Laboratories Agency - Weybridge and CPHL Colindale for their assistance in identification of the isolates.
Ashraf A. F. Elsaghir and Edward A. James

Department of Medical Microbiology, Barnet and Chase Farm Hospitals NHS Trust, Barnet Hospital, Wellhouse Lane, Barnet, Herts EN5 3DJ, UK

Correspondence: Ashraf A. F. Elsaghir (Ashraf.ElSagir@barnet-chase-tr.nhs.uk)

Barham, W. B., Church, P., Brown, J. E. \& Paparello, S. (1993). Misidentification of Brucella species with use of rapid bacterial identification systems. Clin Infect Dis 17, 1068-1069.

Batchelor, B. I., Brindle, R. J., Gilks, G. F. \& Selkon, J. B. (1992). Biochemical misidentification of Brucella melitensis and subsequent laboratory-acquired infections. J Hosp Infect 22, 159-162.

Chang, H. J., Christenson, J. C., Pavia, A. T. \& 11 other authors (1996). Ochrobactrum anthropi meningitis in pediatric pericardial allograft transplant recipients. J Infect Dis 173, 656-660.

Cieslak, T. J., Robb, M. L., Drabick, C. J. \&
Fischer, G. W. (1992). Catheter-associated sepsis caused by Ochrobactrum anthropi: report of a case and review of related nonfermentative bacteria. Clin Infect Dis 14, 902-907.

El-Zimaity, D., Harrison, G. A., Keen, A. P., Price, S., Evans, S. E., Lewis, A. M., Thomas, I., Bevan, V. \& Djemal, K. (2001). Ochrobactrum anthropi pseudobacteraemia. J Infect 43, 217-218.

Ezzedine, H., Mourad, M., Van Ossel, C. \& 7 other authors (1994). An outbreak of Ochrobactrum anthropi bacteraemia in five organ transplant patients. J Hosp Infect 27, 35-42.

Saeed Mahmood, M., Sarwari, A. R., Khan, M. A., Sophie, Z., Khan, E. \& Sami, S. (2000). Infective endocarditis and septic embolization with Ochrobactrum anthropi: case report and review of literature. J Infect 40, 287-290.

Yu, W. L., Lin, C. W. \& Wang, D. Y. (1998).

Clinical and microbiologic characteristics of Ochrobactrum anthropi bacteremia. J Formos Med Assoc 97, 106-112. 